\title{
Specifying I/O using Abstract Nested Hoare Triples in Separation Logic
}

\author{
Willem Penninckx \\ imec-DistriNet, Department of \\ Computer Science \\ KU Leuven \\ Leuven, Belgium \\ willem@willemp.be
}

\author{
Amin Timany \\ imec-DistriNet, Department of \\ Computer Science \\ KU Leuven \\ Leuven, Belgium \\ amin.timany@cs.kuleuven.be
}

\author{
Bart Jacobs \\ imec-DistriNet, Department of \\ Computer Science \\ KU Leuven \\ Leuven, Belgium \\ bart.jacobs@cs.kuleuven.be
}

\begin{abstract}
We propose a separation logic-based approach for modular specification and verification of the I/O behavior of a program. The approach uses higher-order separation logic predicates to express abstract nested Hoare triples that abstractly associate a precondition and a postcondition with an I/O action. The approach supports verifying higher-level $\mathrm{I} / \mathrm{O}$ actions built on top of lower-level ones (e.g. the I/O abstractions offered by the programming language's standard library, implemented on top of system calls), as well as virtual I/O actions that in fact only manipulate memory, against specifications that are indistinguishable from those of the "primitive I/O actions".
\end{abstract}

CCS Concepts • Theory of computation $\rightarrow$ Program specifications; Pre- and post-conditions; Program verification;

Keywords input/output, modular program verification, module specifications, separation logic

\section{ACM Reference Format:}

Willem Penninckx, Amin Timany, and Bart Jacobs. 2019. Specifying I/O using Abstract Nested Hoare Triples in Separation Logic. In Formal Techniques for Java-like Programs (FTffP'19), Fuly 15, 2019, London, United Kingdom. ACM, New York, NY, USA, 7 pages. https: //doi.org/10.1145/3340672.3341118

\section{The Problem of Specifying I/O Behavior}

We introduce the problem addressed by this paper, as well as our proposed approach to address it, in the context of our VeriFast $[3,10]$ approach and tool for modular formal

\footnotetext{
Permission to make digital or hard copies of all or part of this work for personal or classroom use is granted without fee provided that copies are not made or distributed for profit or commercial advantage and that copies bear this notice and the full citation on the first page. Copyrights for components of this work owned by others than ACM must be honored. Abstracting with credit is permitted. To copy otherwise, or republish, to post on servers or to redistribute to lists, requires prior specific permission and/or a fee. Request permissions from permissions@acm.org.

FTf JP'19, Fuly 15, 2019, London, United Kingdom

(c) 2019 Association for Computing Machinery.

ACM ISBN 978-1-4503-6864-3/19/07 .. \$15.00

https://doi.org/10.1145/3340672.3341118
}

verification of $\mathrm{C}$ programs. ${ }^{1}$ VeriFast takes as input a $\mathrm{C}$ compilation unit, annotated with function specifications, as well as other specification constructs such as abstract predicates that describe the layout of data structures and proof hints such as loop invariants, written in a variant of separation logic [6] inside specially marked comments. It performs symbolic execution of each function, starting from a symbolic state that represents an arbitrary state that satisfies the precondition, and checking at every return point that the symbolic state satisfies the postcondition. Symbolic execution of function calls uses the callee's specification, not its implementation. If the tool reports "0 errors found" for all compilation units of a program, then, barring bugs in the tool, and assuming that the specifications provided for the system APIs used by the program are sound, all possible executions of the program are free of undefined behavior and comply with the stated specifications.

In this paper, we consider the problem of modular verification of the interactive $(\mathrm{I} / \mathrm{O})$ behavior of programs such as the following:

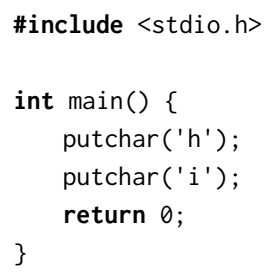

This program uses the putchar function from the $\mathrm{C}$ standard library, declared in header file stdio.h, to output the message "hi" to the standard output stream. However, the default version of stdio.h currently shipping with VeriFast does not allow us to specify this: its specification for putchar is simply

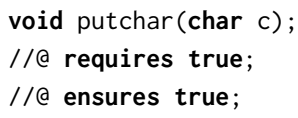

allowing us to verify the specification

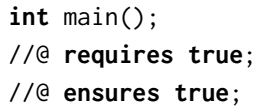

${ }^{1}$ VeriFast supports Java as well. 
for our program, the same specification satisfied also by the program that outputs "bye", or nothing at all.

\section{Abstract Nested Hoare Triples}

\subsection{Basic Idea}

Our proposed approach for specifying and verifying the $\mathrm{I} / \mathrm{O}$ behavior of programs in separation logic is to associate a higher-order separation logic predicate with each I/O action, which we call its transition predicate. We use an I/O action's transition predicate to abstractly associate preconditionpostcondition pairs with the I/O action. In this paper, we adopt the convention of naming a transition predicate after its associated I/O action, with an underscore appended at the end. For example, the assertion putchar $(P, C, Q)$ uses the transition predicate putchar_ to associate precondition $\mathrm{P}$ and postcondition $Q$ with the action of outputting the character $\mathrm{c}$. Here, $P$ and $Q$ are values of type predicate(), i.e. they are themselves separation logic predicates. Notice that such assertions are in fact abstract versions of nested Hoare triples [9]; therefore, we call them abstract nested Hoare triples. We can use them to specify function putchar very abstractly as follows:

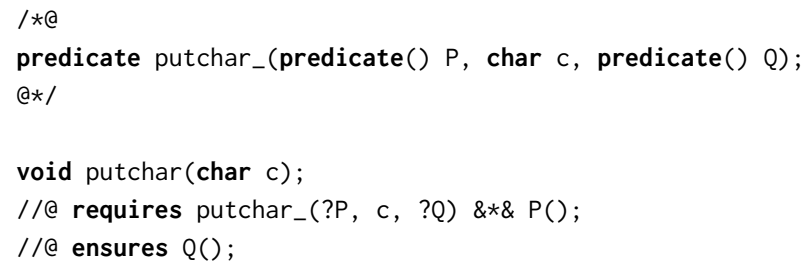

Here, ?P is VeriFast syntax for introducing a logical variable $P$, and $\& * \&$ is the separating conjunction. The scope of a logical variable introduced in a function specification's precondition is the entire specification, i.e. the precondition and the postcondition. Logical variables introduced in the precondition are universally quantified at the level of the specification. That is, the meaning of the above specification is that for any predicates $P$ and $Q$, if putchar_ $(P, C, Q)$ and (separately) $P$ hold in a given state, then putchar (c) may be called, and after such call, putchar_ $(P, C, Q)$ and (some resources described by) $P()$ will have been consumed and (some resources described by) Q() will have been produced.

We can now specify our example program as follows:

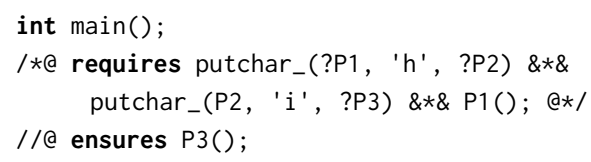

According to this specification, function main starts out with (some resources described by) P1(). Per transition predicate putchar_(P1, ' ' $h$ ', P2), this allows it to output ' $h$ '. (It does not allow it to output ' $i$ ' at this point, since to do so it would need P2(), which it does not yet have.) By outputting ' $h$ ', it loses P1() but gains P2(). Then, by outputting ' $i$ ', it loses P2() and gains $\mathrm{P} 3($ ), which allow it to satisfy its postcondition and terminate.

Notice that this specification expresses the desired property: it states that main shall only ever output a prefix of "hi", and furthermore, that if it returns, it shall have outputted exactly "hi".

Note: we will sometimes refer to the predicates used as the precondition or postcondition argument of transition predicates, such as the predicates $\mathrm{P} 1, \mathrm{P} 2$, and $\mathrm{P} 3$ in the specification of main above, as places, and to assertions that assert such a place, such as $\mathrm{P} 1()$ and $\mathrm{P} 3()$ in the specification of main, as tokens; indeed, the precondition of main can be read as specifying a Petri net with a transition from place $\mathrm{P} 1$ to P2 labelled by action putchar (' $h$ ') and a transition from P2 to P3 labelled by action putchar ( $\mathrm{i}$ ' '), and with a single token at place P1. Firing the two transitions causes the token to move from place P1 to place P3. However, the reader can ignore this analogy if they do not find it useful.

\subsection{Building Higher-Level Actions}

This approach straightforwardly supports defining higherlevel I/O actions on top of lower-level ones. For example, consider the function putc_beep, defined in terms of putchar and some imagined $\mathrm{I} / \mathrm{O}$ action beep:

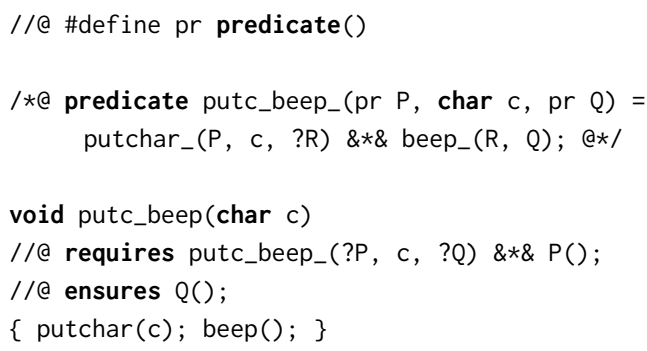

Notice that, even though function putc_beep is not a primitive $\mathrm{I} / \mathrm{O}$ action, clients cannot tell this from its specification; its form is exactly analogous to that of putchar. (Note: the syntax ?R in a predicate definition introduces an existentially quantified logical variable. The definition means: putc_beep_ $(P, C, Q)$ holds if and only if there exists a predicate $R$ such that putchar_ $(P, C, R)$ holds and, separately, beep_( $(R, Q)$ holds.)

\subsection{Underspecification}

Besides elegantly supporting transparently building higherlevel I/O operations on top of lower-level ones, this approach has the additional feature of elegantly supporting underspecification, in two forms: in the form of alternative paths of transition predicates between two places (predicates) $P$ and $Q$, and in the form of enabling multiple concurrent paths of transition predicates by splitting and joining places. In the following example, we use both forms to express that the program may print either "hi!" or "hey!", and that furthermore, the program must beep at some point after printing the "h" and before printing the "!": 


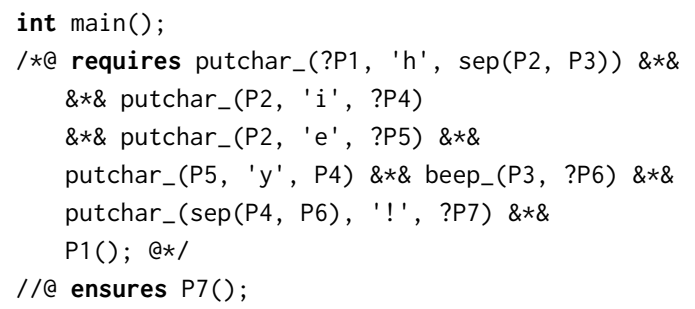

where $\operatorname{sep}(P, Q)$ denotes the separating conjunction of predicates $P$ and $Q$, as a value of type predicate(). (VeriFast does not support directly writing $P() \& * \& Q()$ in a value position.) (To continue the Petri net analogy, a transition predicate whose precondition/postcondition is of the form $\operatorname{sep}(\mathrm{P} 1, \mathrm{P} 2)$ can be thought of as a transition with multiple incoming/outgoing arrows, consuming/producing a token at each incoming/outgoing place.)

\subsection{Input}

Another feature of the approach is elegant support for specifying input actions, and specifying how a program's behavior should depend on the values retrieved from the environment. Furthermore, it supports expressing assumptions about such values, as illustrated by the following example:

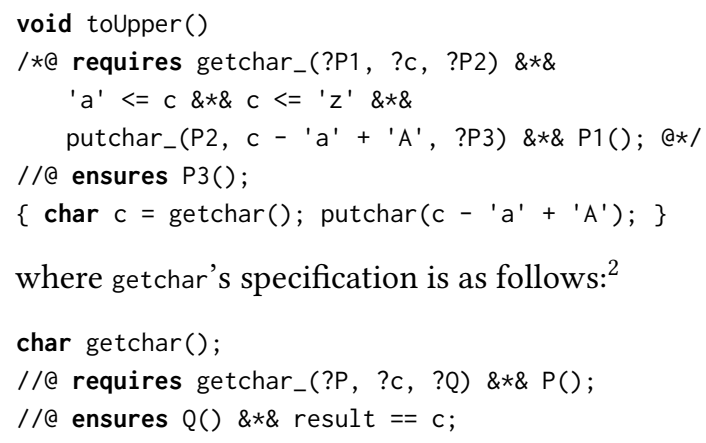

Notice that in this approach, a function's precondition may refer to the values yielded by input actions that the program has yet to perform; this can be thought of as a form of prophecy variables. For example, in the precondition of getchar, logical variable $\mathrm{c}$ refers to the character that the program will receive from the environment when it calls getchar.

The approach we propose here has evolved from earlier work [7]. That earlier work supports the features shown so far, but it does not support the features we show in the remainder of this paper.

\section{$2.5 \quad \mathrm{I} / \mathrm{O}$ and Memory}

Our approach supports transparently implementing higherlevel I/O operations in terms of a combination of lower-level I/O operations and memory manipulation. For example, consider the following implementation of the flush and putchar

\footnotetext{
${ }^{2}$ The real getchar function returns an int and may report failure; for simplicity, we ignore these complications here.
}

C standard library functions ${ }^{3}$ in terms of the write system call:

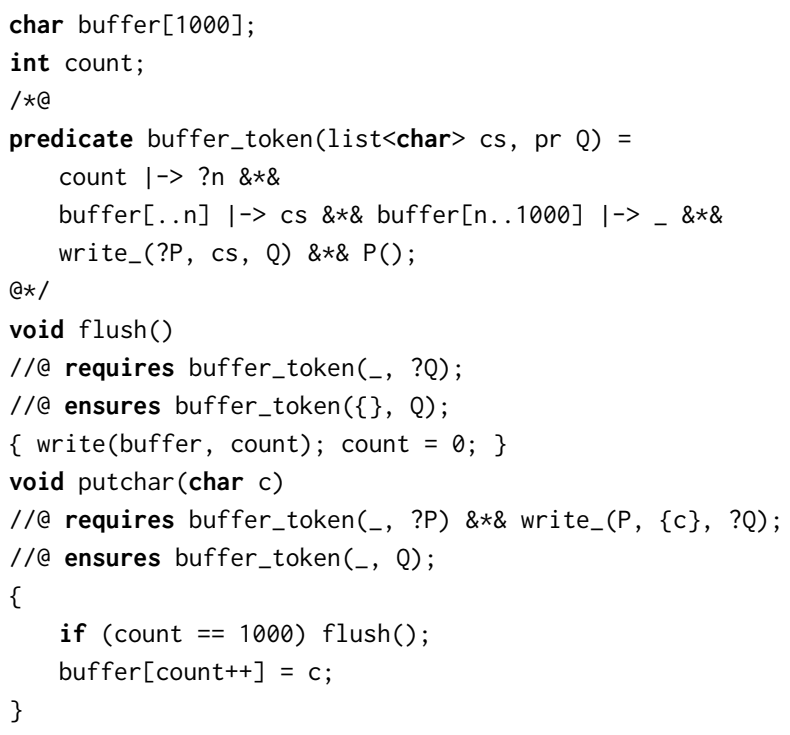

( \{\} and $\{c\}$ are VeriFast syntax for the empty list and the singleton list containing character c, respectively.) We can verify these functions against the stated specifications, if we assume the following (simplified) declaration of write:

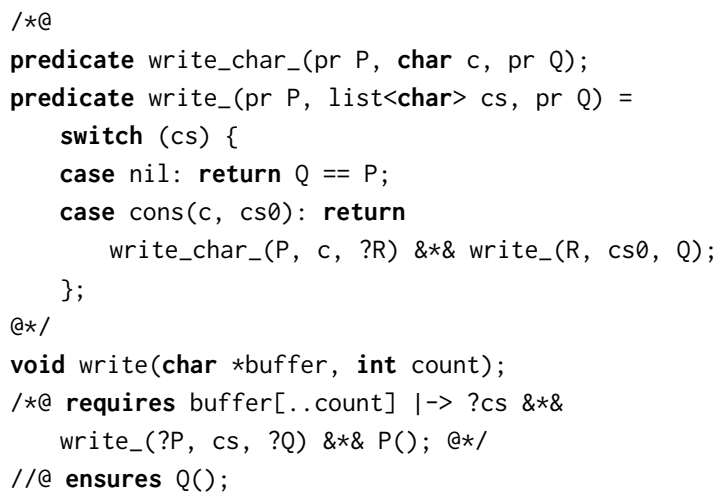

The predicate buffer_token(cs, Q) asserts ownership of the global variables count and buffer, as well as a token $P()$ that allows the program to obtain a token at $Q$ (i.e. to obtain $Q()$ ) by writing the contents cs of the buffer. The specification for putchar states that, given a token that allows the program to reach $Q$ after flushing the pre-state buffer and then writing $c$, it produces a token that allows the program to reach $Q$ after flushing the post-state buffer.

This specification is fine, except that it is different from the specification for putchar we saw earlier. The specification we really want is the following:

void putchar (char $\mathrm{c}$ );

//@ requires putchar_(?P, c, ?Q) \&*\& $P()$; //@ ensures $Q()$;

\footnotetext{
${ }^{3}$ This is a simplification; the real function is called fflush and takes the
} stream to be flushed as an argument. 
Fortunately, however, we can match the two specifications by defining putchar_appropriately:

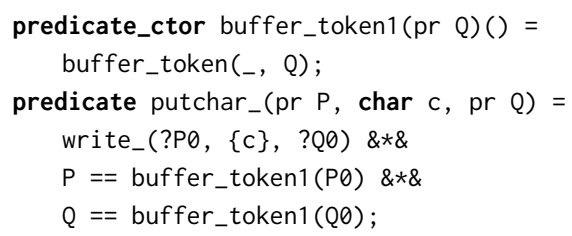

(The predicate constructor buffer_token1 allows the assertion buffer_token(_, Q), for some particular Q, to be used as a value of type predicate().) In fact, we can make any function specification

void foo(int arg);

//a requires $\mathrm{P}(\arg , ? \mathrm{x})$;

// ensures $Q(x)$;

match an "I/O-style" specification

void foo(int arg)

//@ requires foo_(?P1, arg, ?Q1) \&*\& P1();

//@ ensures Q1();

by defining the transition predicate foo_ as

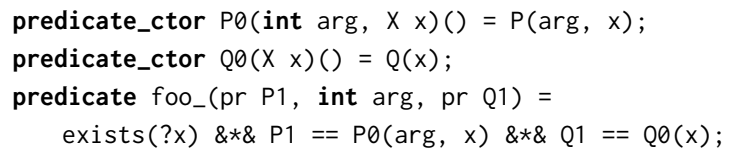

Similarly, we can prove an I/O-style specification for flush:

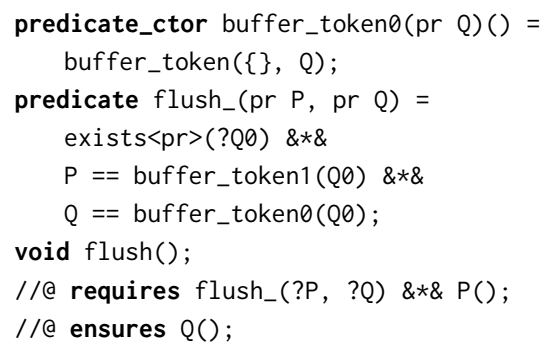

We can now verify a client program of putchar and flush in a way that is completely agnostic to the way these functions are implemented:

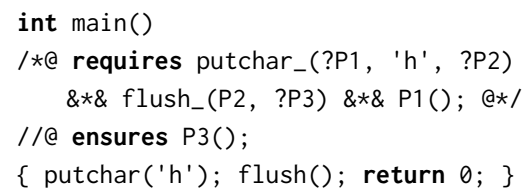

and then use this proof to verify a lower-level specification of the program in terms of system calls:

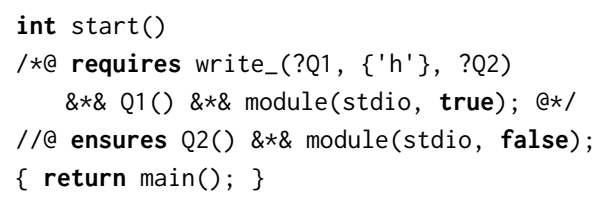

(In some C implementations on Linux, a program's start function is its actual low-level entry point. It is usually auto-generated by the $\mathrm{C}$ compiler. It first initializes the standard library and then calls the main function.) (The assertion module(M, init) asserts ownership of the global variables of module $M$, either initialized to zero if init is true, or in an arbitrary state otherwise.)

\subsection{Mixing Abstraction Levels}

Consider now a variant of the above program, where main uses both the high-level putchar operation and the low-level beep operation:

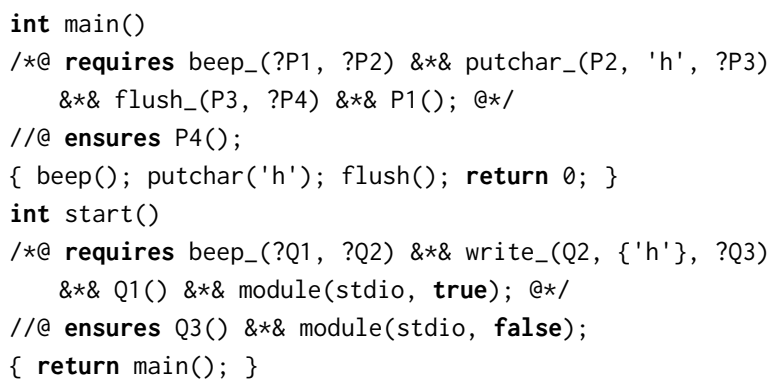

Notice that we cannot verify the call of main in start: to prove beep_(P1, P2) we only have beep_(Q1, Q2) available; therefore, we must instantiate $\mathrm{P} 1$ with Q1 and P2 with Q2. However, the unknown predicate Q2 is not (necessarily) of the form buffer_token1(_), as required by putchar_. In fact, we know Q1 (and therefore Q2) does not include ownership of the global variables count and buffer, since we have Q1() and separately module(stdio, true). However, putchar needs access to these variables.

Notice, however, that we can solve this problem if we can assume a frame axiom for transition predicate beep_:

$$
\text { beep_(Q1, Q2) } \Rightarrow \text { beep_(sep(Q1, ebuf), } \operatorname{sep}(Q 2 \text {, ebuf)) }
$$

similar to the Frame Rule of separation logic, as applied to function call beep():

$$
\frac{\{\text { Frame }}{\{\mathrm{Q} 1\} \text { beep }()\{\mathrm{Q} 2\}}
$$

where ebuf() asserts ownership of the empty buffer:

predicate ebuf ()$=$ count $|-\rangle 0 \& * \&$ buffer[..1000] $|-\rangle_{-}$;

To enable this type of scenario, we therefore introduce a convention that each transition predicate shall be accompanied by such a frame axiom. In VeriFast, this takes the form of a lemma function, a type of ghost function:

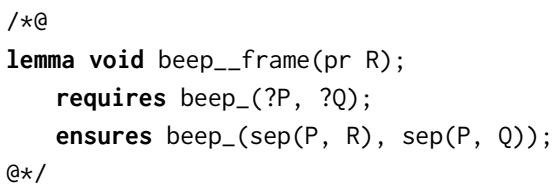


This lemma almost allows us to verify function start above. The only problem is that while $\operatorname{sep}(\mathrm{Q} 2$, ebuf) implies buffer_token1(Q2), it is not equivalent and hence not equal to it. ${ }^{4}$ To complete the proof, we also need a weakening property:

$$
\frac{\mathrm{P} 0 \Rightarrow \mathrm{P} 1 \quad \mathrm{Q} 0 \Rightarrow \mathrm{Q} 1}{\text { beep_(P1, Q } 0)^{2} \text { beep_(P0, Q1) }}
$$

analogous to Hoare logic's Rule of Consequence applied to a beep() call:

$$
\begin{aligned}
& \text { Conseq } \\
& \frac{\mathrm{P} 0 \Rightarrow \mathrm{P} 1 \quad\{\mathrm{P} 1\} \text { beep }()\{\mathrm{Q} 0\} \quad \mathrm{Q} 0 \Rightarrow \mathrm{Q} 1}{\{\mathrm{P} 0\} \text { beep }()\{\mathrm{Q} 1\}}
\end{aligned}
$$

So, by also introducing the convention that each transition predicate shall be accompanied by a weakening axiom, we enable support for verifying programs where some function specifications mix I/O actions of different abstraction levels.

Note: we can easily make our definitions of putchar_ and flush_above satisfy the frame and weakening properties as well by adapting them as follows:

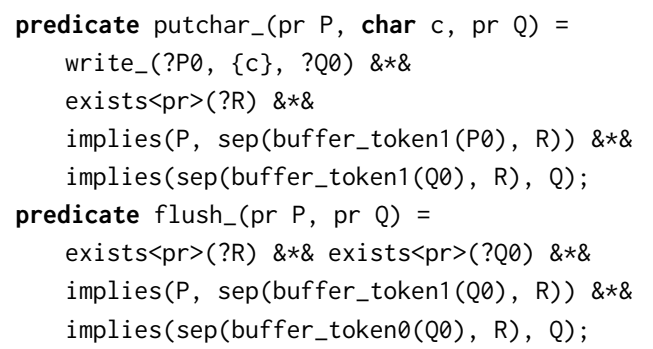

This allows these $\mathrm{I} / \mathrm{O}$ actions to be mixed in function specifications with even higher-level ones.

\subsection{Virtual Input}

Clearly, our approach also supports I/O-style specifications for purely "virtual I/O actions", such as those of Java's ByteArrayOutputStream, which simply appends any "outputted" bytes to an in-memory buffer. A more interesting instance of this is in the context of the verification of multithreaded programs, where threads communicate through shared queues. One might want to verify each thread in such a way that their proof can be reused unchanged if one later decides to run the threads in separate processes or on separate machines, communicating via sockets. Our approach enables this, by enabling I/O-style specifications for the shared queue operations; these can then later be replaced transparently by actual I/O operations, such as socket operations.

Notice, however, that in our proposed specification style for input operations, as illustrated by the specification of getchar above, the input operation's transition predicate takes the operation's result as an argument. In general, to construct a definition for such a transition predicate for a virtual input operation, prophecy variables are needed. Consider for

\footnotetext{
${ }^{4}$ In fact, in VeriFast predicate extensionality does not hold, so the predicate values would not be equal even if they were equivalent.
}

example a (contrived) implementation of getchar in terms of a random number generator: ${ }^{5}$

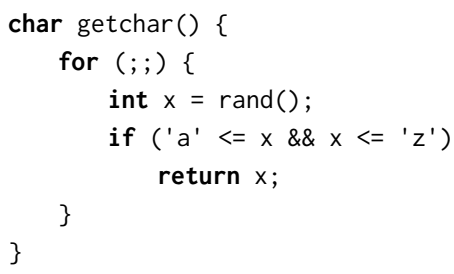

where the specification of rand() is:

int $\operatorname{rand}()$;

//@ requires true;

// @ ensures true;

This implementation of getchar returns some arbitrary lowercase letter. We now want to verify the following program:

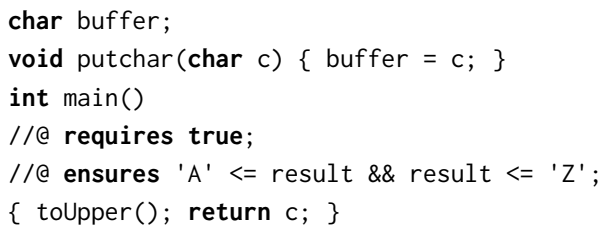

Here, we use function toupper specified and verified above.

In order to be able to reuse the existing proof of toupper in the proof of this program, we need to verify our implementation of getchar against the specification shown above. We can do so by using an encoding of prophecy variables into VeriFast as follows:

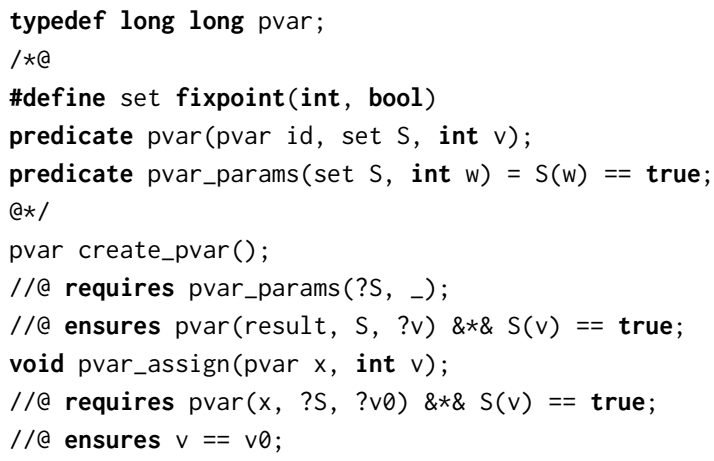

This encoding defines a function for creating a prophecy variable and a function for assigning a value to a prophecy variable created earlier. When creating a prophecy variable, a set of integers $S$ and a witness w showing that the set is nonempty have to be supplied. The operation produces a predicate $\operatorname{pvar}(x, s, v)$ asserting that $x$ is the identifier of a prophecy variable constrained by set $\mathrm{s}$ and with prophecized value $\mathrm{v}$, which is in set $\mathrm{s}$. Assigning the prophecy variable consumes the pvar predicate and requires that the supplied value $v$ is in the set $S$, and produces the fact that the supplied value equals the prophecized value.

\footnotetext{
${ }^{5} \mathrm{~A}$ more realistic example, of a multithreaded chat server where prophecy variables are needed to deal with nondeterminism caused by the interleaving of threads, is offered in the accompanying technical report of this paper [8].
} 
Note that the functions create_pvar and pvar_assign are declared in this VeriFast encoding as real functions, not as ghost functions, even though they are in fact no-ops at run time. This is necessary for soundness: if pvar_assign was a ghost operation, we could $v+1$ to the prophecy variable, where $v$ is the prophecized value, leading to the contradiction $v+1==v$. To eliminate this hack, we plan to equip VeriFast with support for multiple levels of ghost code, to ensure that the prophecized value is "more ghost" than the prophecy variable assignment operation.

We can now prove the example program as follows, using the frame properties for getchar_ and putchar_ to frame off ownership of global variables $p$ and buffer, respectively:

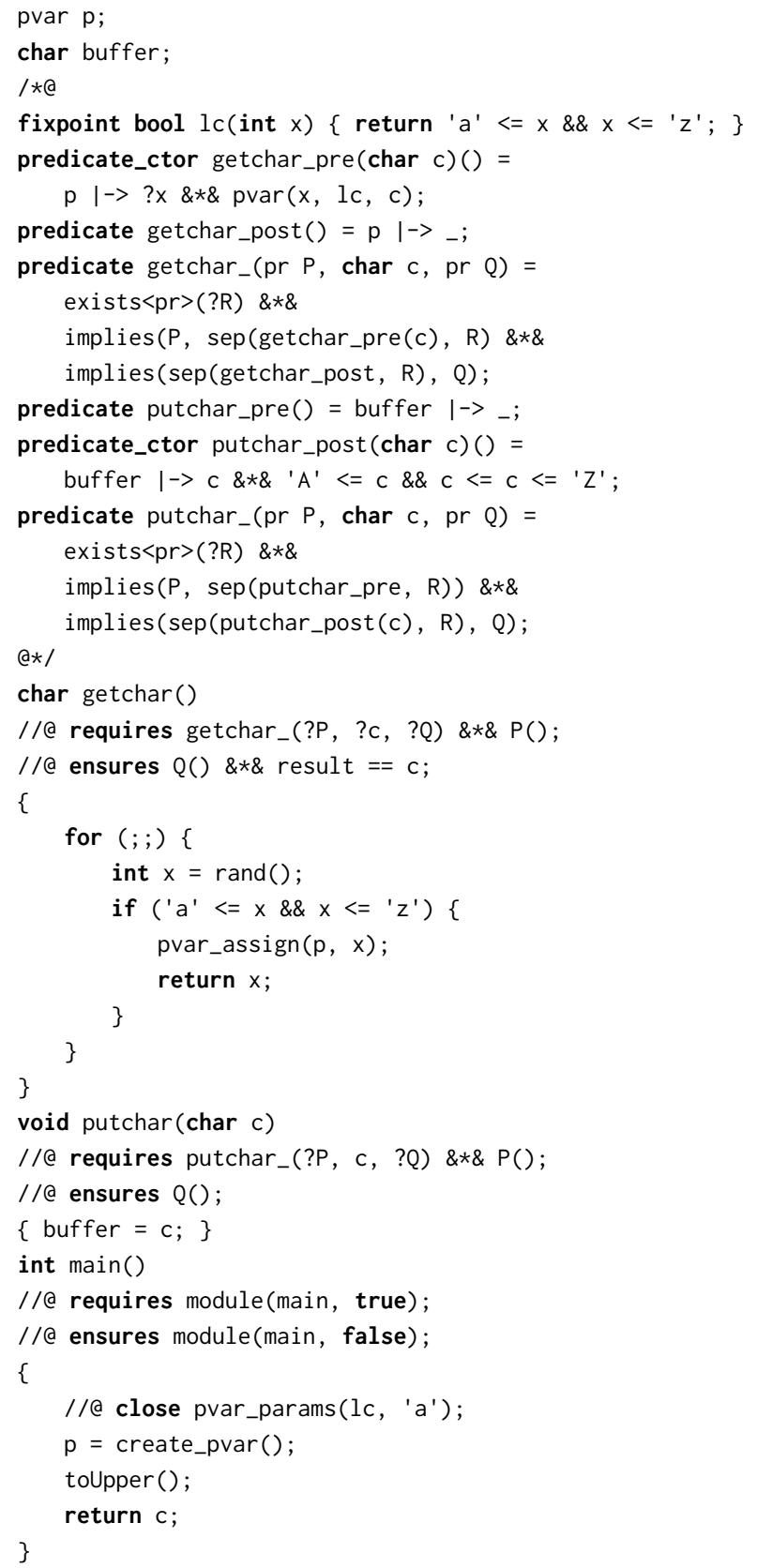

\section{Related Work}

Our earlier work [7] proposed a specification style for I/O based on notions of Petri nets and tokens built into the logic. In that logic, I/O-style specifications cannot be interpreted as predicates about memory, preventing the use of $\mathrm{I} / \mathrm{O}$-style specifications for semi-virtual (e.g. buffered) or fully virtual I/O operations.

Férée et al. [1] performed a separation-logic based machinechecked verification of a number of classical UNIX commandline tools largely implemented as shallowly embedded functions in HOL4. Their specifications for the I/O operations are in terms of a separation logic predicate STDIO(fs) where fs reflects the state of the file system.

Ho et al. [2] propose an approach for proof-producing synthesis of imperative programs with $\mathrm{I} / \mathrm{O}$ from monadic functions of higher-order logic. For reasoning about I/O, they use the same approach as Férée et al..

Koh et al. [4] used the Verified Software Toolchain to produce a machine-checked separation logic-based proof of the functional correctness of a simple networked server written in C. In their approach, specifications of I/O operations are in terms of a separation logic predicate ITree $(t)$ where $\mathrm{t}$ is an interaction tree, a coinductive (i.e. lazy) tree of $\mathrm{I} / \mathrm{O}$ operations and (angelic) choice points. Branching occurs on choice points and on the result values (i.e. inputs) yielded by I/O operations. Their trees do not seem to be able express assumptions about the environment.

\section{Conclusion}

In this short paper, we outlined an approach for modular reasoning about the $\mathrm{I} / \mathrm{O}$ behavior of programs in a VeriFastlike setting. It supports building higher-level I/O operations on top of lower-level ones, mixing I/O operations of varying abstraction levels in function specifications, and treating semi-virtual or fully virtual I/O operations as if they were primitive ones.

The technical report accompanying this paper [8] offers a formal definition of the syntax and semantics of a programming language with $\mathrm{I} / \mathrm{O}$, a formal definition and soundness proof of the proposed logic, and formalized versions of a buffered I/O example and a virtual I/O example involving a multithreaded chat server where threads communicate through a shared queue. The examples shown in the technical report have been formalized both in VeriFast and in Coq; for the latter, we used the Iris [5] library for separation logic reasoning about concurrent higher-order programs.

Note: the technical report proposes a formalization of prophecy variables. We explicitly do not claim that part as a contribution of this paper; we are preparing a paper on that topic for submission elsewhere. 


\section{References}

[1] Hugo Férée, Johannes Åman Pohjola, Ramana Kumar, Scott Owens, Magnus O. Myreen, and Son Ho. 2018. Program Verification in the Presence of I/O - Semantics, Verified Library Routines, and Verified Applications. In Verified Software. Theories, Tools, and Experiments 10th International Conference, VSTTE 2018, Oxford, UK, Fuly 18-19, 2018, Revised Selected Papers (Lecture Notes in Computer Science), Ruzica Piskac and Philipp Rümmer (Eds.), Vol. 11294. Springer, 88-111. https: //doi.org/10.1007/978-3-030-03592-1_6

[2] Son Ho, Oskar Abrahamsson, Ramana Kumar, Magnus O. Myreen, Yong Kiam Tan, and Michael Norrish. 2018. Proof-Producing Synthesis of CakeML with $\mathrm{I} / \mathrm{O}$ and Local State from Monadic HOL Functions. In Automated Reasoning - 9th International foint Conference, IFCAR 2018, Held as Part of the Federated Logic Conference, FloC 2018, Oxford, UK, July 14-17, 2018, Proceedings (Lecture Notes in Computer Science), Didier Galmiche, Stephan Schulz, and Roberto Sebastiani (Eds.), Vol. 10900 Springer, 646-662. https://doi.org/10.1007/978-3-319-94205-6_42

[3] Bart Jacobs (Ed.). 2018. VeriFast 18.02. Zenodo. https://doi.org/10. 5281/zenodo.1182724

[4] Nicolas Koh, Yao Li, Yishuai Li, Li-yao Xia, Lennart Beringer, Wolf Honoré, William Mansky, Benjamin C. Pierce, and Steve Zdancewic. 2019. From $C$ to interaction trees: specifying, verifying, and testing a networked server. In Proceedings of the 8th ACM SIGPLAN International Conference on Certified Programs and Proofs, CPP 2019, Cascais, Portugal, fanuary 14-15, 2019, Assia Mahboubi and Magnus O. Myreen (Eds.). ACM, 234-248. https://doi.org/10.1145/3293880.3294106

[5] Robbert Krebbers, Ralf Jung, Ales Bizjak, Jacques-Henri Jourdan, Derek Dreyer, and Lars Birkedal. 2017. The Essence of Higher-Order Concurrent Separation Logic. In ESOP.

[6] Peter W. O'Hearn, John C. Reynolds, and Hongseok Yang. 2001. Local Reasoning about Programs that Alter Data Structures. In Computer Science Logic, 15th International Workshop, CSL 2001. 10th Annual Conference of the EACSL, Paris, France, September 10-13, 2001, Proceedings (Lecture Notes in Computer Science), Laurent Fribourg (Ed.), Vol. 2142. Springer, 1-19. https://doi.org/10.1007/3-540-44802-0_1

[7] Willem Penninckx, Bart Jacobs, and Frank Piessens. 2015. Sound, Modular and Compositional Verification of the Input/Output Behavior of Programs. In ESOP.

[8] Willem Penninckx, Amin Timany, and Bart Jacobs. 2019. Abstract I/O Specification. CoRR abs/1901.10541 (2019). arXiv:1901.10541 http: //arxiv.org/abs/1901.10541

[9] Jan Schwinghammer, Lars Birkedal, Bernhard Reus, and Hongseok Yang. 2011. Nested Hoare triples and frame rules for higher-order store. Logical Methods in Computer Science 7, 3 (2011).

[10] Frédéric Vogels, Bart Jacobs, and Frank Piessens. 2015. Featherweight VeriFast. Logical Methods in Computer Science 11, 3 (2015). https: //doi.org/10.2168/LMCS-11(3:19)2015 